\section{Nivel de ansiedad a los factores ambientales en dos servicios odontológicos públicos: hospitalario y universitario}

\author{
Anxiety level related to environmental factors in two public \\ dental services: hospital and universitary
}

\begin{abstract}
Resumen
El objetivo del estudio fue comparar los niveles de ansiedad generados en los pacientes que acuden a un consultorio odontológico en dos servicios públicos. Se obtuvieron datos de pacientes que hayan acudido por lo menos una vez en un ańo calendario a un servicio odontológico, la ansiedad se midió con el test de Corah. La muestra estuvo conformada por 100 pacientes (50 de cada servicio). Se obtuvo que el $39 \%$ de los pacientes presentó ansiedad moderada, un $19 \%$ en el servicio universitario y un $20 \%$ en el servicio hospitalario; el $15 \%$ de los pacientes presentó ansiedad severa, un $9 \%$ en el hospitalario y un $6 \%$ en el servicio universitario. Mayor porcentaje de ansiedad moderada, alta y severa se presentó en el servicio hospitalario. Otra sección de la encuesta fue la Evaluación de los Miedos Dentales. Se concluye que el tiempo y el costo que demanda la atención son los factores que causan más ansiedad en los pacientes.

Palabras clave: ansiedad, ambiente, atención odontológica
\end{abstract}

\section{Abstract}

The aim of this study was to compare the levels of dental anxiety in patients who visited dental offices in two public services. Information was obtained from patients who had visited their dentists at least once the present year. The sample size was 100 patients (50 per service). Applying the Corah's Dental Questionnaire, it was established that $39 \%$ of patients presented moderate anxiety, $19 \%$ in universitary service and $20 \%$ in hospital service; $15 \%$ of the patients presented severe anxiety, $9 \%$ in hospital service and $6 \%$ in universitary service. A great percentage of moderate, high and severe anxiety was perceived in hospital service. Applying the Dental Concerns Assessment, it was concluded that time and costs invested on dental attention are the factors that cause more anxiety in patients among others considered.

Key words: anxiety, environment, dental care
Artículo Original
Jean Paul Cirilo Jacobo ${ }^{1}$, Edin
Majuán Córdova ${ }^{1}$, Yazmín
Marín Flores ${ }^{1}$, Mary Flor
Robles $^{1}$, Kariem Siquero
Vera $^{1}$, Ana María Díaz Soriano

1 Estudiantes. Facultad de Odontología. Universidad Nacional Mayor de San Marcos.

2 Docente Dpto. Académico Estomatología Preventiva y Social. Facultad de Odontología. Universidad Nacional Mayor de San Marcos.

\section{Correspondencia:}

Mg. Ana María Díaz Soriano

Av. Germán Amézaga s/n

Facultad Odontología UNMSM. Ciudad Universitaria

Teléfono: 619-7000 anexo 3405

Correo electrónico: anamards@yahoo.es

Fecha de recepción: 19-12-11

Fecha de aceptación: 28-03-12

\section{Introducción}

El nivel de ansiedad del paciente en una atención odontológica frente a los factores ambientales, aún no es estudiado en el Perú. La ansiedad es una característica enlazada con la actividad humana, y no debe ser aislada de la profesión odontológica. La investigación analiza las diferencias entre el nivel de ansiedad de dos servicios públicos de atención odontológica, uno hospitalario y otro universitario, específicamente respecto a los factores ambientales, que generan ansiedad en los pacientes. La ansiedad dental se describe como aprehensión más profunda que interfiere con el tratamiento rutinario y requiere una atención especial. El campo ambiental, considera cuatro factores odontológicos: limpieza, olores, sonidos y tiempo de espera.

En relación con la interacción paciente-odontólogo, Rojas y Misrachi Launert ${ }^{1}$ refieren en su investigación la importancia de la interacción y su influencia en el desarrollo de la ansiedad. Es necesario seguir desarrollando investigaciones que aumenten el conocimiento en esta área para disponer de mejores herramientas en la relación dentista-paciente a modo de favorecer un mejor nivel de salud oral en la población. Méndez ${ }^{2}$ menciona la ansiedad en los seres humanos, atribuida a situaciones específicas y la ayuda para enfrentarla; pero cuando esta se eleva puede llegar a ser perjudicial para el organismo y su desempeño durante la atención dental. Al tener una menor experiencia en la consulta con pacientes revela niveles de ansiedad muy elevados que pueden llegar a ser incontrolables y de alguna manera bloquean el desempeño pleno de sus actividades; mientras que en odontólogos con mayor experiencia los pacientes presentan niveles de ansiedad normales. De aquí la importancia del manejo de la ansiedad por parte del odontólogo para una atención dental adecuada.

Los resultados obtenidos de diversas investigaciones demuestran que los odontólogos desconocen el área psicológica de la atención odontológica, lo que influye en el tratamiento que realizan; los odontólogos no cuentan con medidas para disminuir los niveles de ansiedad de sus pacientes, tomando en cuenta que aproximadamente el $95 \%$ de la población peruana padece o ha padecido de caries dental y/o enfermedad periodontal, es necesario determinar la influencia de la ansiedad en la negativa de los pacientes a recibir atención odontológica.

La ansiedad debida a los factores ambientales (limpieza, olores, ruidos, tiempo de espera) ha sido considerada por muchos autores como parte de un todo; sin embargo, no se ha evaluado a este conjunto de factores ambientales 
como un poderoso agente ansiógeno por sí mismo. El objetivo del estudio es comparar los factores ambientales desencadenantes de ansiedad en pacientes que acuden a un servicio odontológico hospitalario y a uno universitario.

\section{Material y método}

Es un estudio observacional, analítico y transversal. La muestra estuvo compuesta por 100 pacientes: 50 de ellos acudían a consulta odontológica en el Hospital Arzobispo Loayza, Lima; y 50, en la Clínica Post Grado de la Universidad Nacional Mayor de San Marcos. Estos fueron seleccionados mediante un muestreo no probabilístico por conveniencia y previo consentimiento informado. Se tomaron en cuenta algunos criterios de inclusión: ser del servicio y encontrarse en la sala de recepción del consultorio hospitalario o universitario y haber asistido a consulta odontológica por lo menos una vez en el presente año. Solo se encuestó a personas mayores de 17 ańos y menores de 80 años.

El instrumento de recolección de datos fue el cuestionario dental de Corah, se midió con la Escala de Ansiedad de Corah DAS-R (Dental Anxiety Scale Revised). El estudio se basó en un método descriptivo comparativo, se usaron las variables cualitativas ordinales para hallar el nivel de ansiedad según el valor numeral que arrojó la encuesta, el valor se midió según el patrón de niveles de ansiedad.

En la descripción de los resultados se utilizó porcentaje ya que la totalidad de la muestra fue 100 y las frecuencias absolutas y los porcentjes fueron coincidentes.

Una vez realizadas las encuestas, se procedió con su análisis estadístico mediante el paquete estadístico SPSS versión 19.

\section{Resultados}

Se halló que los pacientes presentan principalmente ansiedad moderada $y$ baja en similar proporción entre los dos tipos de servicios. La categoría de ansiedad severa que se ubica en tercer lugar de frecuencia predomina ligeramente en el servicio hospitalario.
Tabla 1. Nivel de ansiedad según el puntaje Corah en el servicio universitario y hospitalario

\begin{tabular}{lccc}
\hline \multirow{2}{*}{ Corah Puntaje Total } & \multicolumn{2}{c}{ Servicio de Salud } & \\
\cline { 2 - 3 } & Universitario & Hospitalario & Total \\
\hline Ansiedad baja & 23 & 15 & 38 \\
Ansiedad moderada & 19 & 20 & 39 \\
Ansiedad alta & 2 & 6 & 8 \\
Ansiedad severa (fobia) & 6 & 9 & 15 \\
Total & 50 & 50 & 100 \\
\hline
\end{tabular}

Fuente: Encuesta realizada por el grupo de investigación. Chi Cuadrada 4,62 gl3 p 0,202

Con respecto al factor limpieza la mayoría de los pacientes se sienten relajados (38 \%), de ellos $26 \%$ son del servicio universitario y $12 \%$ del hospitalario. Mientras que fueron pocos los pacientes que se sienten muy ansiosos (4\%), de ellos la mayoría del servicio hospitalario

Tabla 2. Nivel de ansiedad al factor ambiental: Limpieza según el Puntaje Corah en el servicio hospitalario y universitario.

\begin{tabular}{lcccccc}
\hline & \multicolumn{5}{c}{ Escala de Corah Pregunta A } & Total \\
\cline { 2 - 6 } Servicio de Salud & Relajado & $\begin{array}{c}\text { Un poco } \\
\text { intranquilo }\end{array}$ & Tenso & Ansioso & $\begin{array}{c}\text { Muy } \\
\text { ansioso }\end{array}$ & \\
\hline Universitario & 26 & 16 & 4 & 3 & 1 & 50 \\
Hospitalario & 12 & 21 & 3 & 11 & 3 & 50 \\
Total & 38 & 37 & 7 & 14 & 4 & 100 \\
\hline
\end{tabular}

Fuente: Encuesta realizada por el grupo de investigación. Chi Cuadrada 11,548 gl4 p 0,021

Referente a los olores del ambiente, la mayoría de los pacientes se sienten un poco intranquilos, de ellos prevalecen los del servicio universitario y en el siguiente lugar se ubica la categoría de tenso en la cual la mayoría de pacientes son del servicio hospitalario.

Tabla 3. Nivel de ansiedad al factor ambiental: Olores según el Puntaje Corah en el servicio hospitalario y universitario

\begin{tabular}{lcccccc}
\hline & \multicolumn{5}{c}{ Escala de Corah Pregunta B } & \\
\cline { 2 - 6 } Servicio de Salud & Relajado & $\begin{array}{c}\text { Un poco } \\
\text { intranquilo }\end{array}$ & Tenso & Ansioso & $\begin{array}{c}\text { Muy } \\
\text { ansioso }\end{array}$ & \multirow{2}{*}{ Total } \\
\hline Universitario & 14 & 19 & 7 & 7 & 3 & 50 \\
Hospitalario & 12 & 13 & 21 & 3 & 1 & 50 \\
Total & 26 & 32 & 28 & 10 & 4 & 100 \\
\hline
\end{tabular}

Fuente: Encuesta realizada por el grupo de investigación. Chi Cuadrada 10,879 g|4 p 0,028

El mayor porcentaje de los pacientes se sienten tensos respecto al factor ambiental sonido en el servicio universitario. Solamente $8 \%$ se sienten muy ansioso, la mayoría son del servicio hospitalario. 
Tabla 4. Nivel de ansiedad al factor ambiental: sonidos según el Puntaje Corah en el servicio hospitalario y universitario.

\begin{tabular}{lcccccc}
\hline \multirow{2}{*}{ Servicio de Salud } & \multicolumn{5}{c}{ Escala de Corah Pregunta C } & \multirow{2}{*}{ Total } \\
\cline { 2 - 6 } & Relajado & $\begin{array}{c}\text { Un poco } \\
\text { intranquilo }\end{array}$ & Tenso & Ansioso & $\begin{array}{c}\text { Muy } \\
\text { ansioso }\end{array}$ & \\
\hline Universitario & 8 & 13 & 21 & 6 & 2 & 50 \\
Hospitalario & 8 & 14 & 9 & 13 & 6 & 50 \\
Total & 16 & 27 & 30 & 19 & 8 & 100 \\
\hline
\end{tabular}

Chi Cuadrada 9,416 gl4 p 0,052

En relación al factor ambiental / tiempo de espera, la mayoría de pacientes se sienten un poco intranquilos, con mayor frecuencia en el servicio hospitlario. Pocos pacientes se sienten muy ansiosos (6\%), de los cuales la mayoría son del servicio hospitalario $(4 \%)$.

Tabla 5. Nivel de ansiedad al factor ambiental Tiempo de espera según el Puntaje Corah en el servicio hospitalario y universitario

\begin{tabular}{llccccc}
\hline \multirow{2}{*}{ Servicio de Salud } & \multicolumn{5}{c}{ Escala de Corah Pregunta D } & \\
\cline { 2 - 6 } & Relajado & $\begin{array}{c}\text { Un poco } \\
\text { intranquilo }\end{array}$ & Tenso & Ansioso & $\begin{array}{c}\text { Muy } \\
\text { ansioso }\end{array}$ & \\
\hline Universitario & 9 & 16 & 14 & 9 & 2 & 50 \\
Hospitalario & 7 & 18 & 8 & 13 & 4 & 50 \\
Total & 16 & 34 & 22 & 22 & 6 & 100 \\
\hline
\end{tabular}

Chi Cuadrada 3,398 gl4 p 0,494

\section{Discusión}

No se confirmó la hipótesis en la cual el servicio hospitalario presenta un mayor nivel de ansiedad en la evaluación global (Tabla 1). Sin embargo, se hallaron diferencias significativas respecto a los factores específicos como limpieza y olores del ambiente.

La frecuencia de pacientes relajados ante el factor ambiental limpieza es mayor en el servicio asistencial universitario $(26 \%)$ con una diferencia del 14 $\%$ respecto al servicio hospitalario. El $21 \%$ de pacientes que asisten a servicio hospitalario se presentan un poco intranquilos, en el servicio universitario en un $16 \%$. Estos resultados sugieren la mejor conservación de la limpieza de los ambientes de servicio universitario, ya que parte importante de su presentación ante el público es la limpieza del ambiente, para captar un mayor número de usuarios.

El nivel de ansiedad de carácter tenso ante el factor ambiental: olores presenta una diferencia de $14 \%$, siendo mayor en el servicio hospitalario (21\%), re- sultados que se deberían a las deficiencias de zonas y áreas de ventilación en el consultorio odontológico y sala de recepción.

El $30 \%$ de los pacientes se encuentra tenso ante estímulos de sonidos, hay una diferencia del $12 \%$ mayor en el servicio universitario. La principal fuente de ruido son la compresora y los equipos de alta velocidad. En la clínica de Post Grado, las unidades dentales se encuentran separadas por menos de un metro de longitud una de otra, lo cual amplifica la intensidad del ruido en cada Especialidad (Rehabilitación Oral, Endodoncia, Ortodoncia, Periodoncia, Odontopediatría) y contribuye a la difusión de las ondas sonoras en las salas de recepción y espera. En el centro hospitalario, la separación entre una unidad dental y otra es mayor. Otro aspecto importante es el número de citas, que condiciona mayor sensibilidad a los ruidos odontológicos por la frecuencia a la consulta.

El tiempo de espera es, por lo general, mayor en el servicio hospitalario que en el universitario, debido a la falta de recursos humanos y la gran demanda de la población. Los datos obtenidos muestran que, el $34 \%$ de los pacientes se sienten un poco intranquilos. El mismo porcentaje $(22 \%)$ de pacientes de ambos servicios se sienten tensos y ansiosos; sin embargo, el $14 \%$ de los pacientes que se sienten tensos estuvieron en el servicio universitario, y el 13 $\%$ de pacientes que se sienten ansiosos asistieron a un servicio público. Los grados más altos de ansiedad (ansioso y muy ansioso) se dan con más frecuencia en el servicio hospitalario, mientras que los grados más bajos y moderados de ansiedad (relajado y tenso) se dan en el servicio universitario. La ansiedad experimentada depende de la calidad del tiempo de espera. En los servicios públicos hospitalarios, los pacientes esperan desde horas muy tempranas para ser atendidos, en los servicios universitarios, el tiempo de espera es más reducido porque las citas son programadas.

La frecuencia de asistencia a consultas odontológicas brinda un punto de partida para medir la experiencia de los pacientes ante su asistencia a un consultorio odontológico. Ante un mayor número de asistencia a sus citas odontológicas, mayor será la experiencia del paciente, por lo que este tendrá un mejor manejo de su ansiedad. La asistencia a servicio hospitalario es menor por lo que tendrá una menor experiencia y menor manejo de ansiedad.

\section{Conclusiones}

1. Los factores ambientales de un consultorio odontológico ocasionan ligeramente mayor nivel de ansiedad a pacientes del servicio hospitalario en comparación con el servicio universitario, no siendo estadísticamente significativo.

2. El factor ambiental limpieza ocasiona un bajo nivel de ansiedad.

3. El factor ambiental olores provoca intranquilidad con mayor frecuencia en pacientes de servicio universitario y tensión, con mayor frecuencia en pacientes de un servicio hospitalario.

4. El factor ambiental sonidos provoca tensión, con mayor frecuencia en pacientes de servicio universitario $y$, ansiedad con mayor frecuencia en pacientes de servicio hospitalario.

5. El factor de ansiedad tiempo de espera provoca intranquilidad con mayor frecuencia en pacientes de servicio hospitalario. 


\section{Referencias bibliográficas}

1. Rojas Alcayaga G, Misrachi Launert C. La interacción paciente-dentista, a partir del significado psicológico de la boca. Av Odontoestomatol [revista en la Internet]. 2004 Ago [citado2012Ene]; 20(4): 185-191. Disponible en: http:// scielo.isciii.es/scielo.php?script $=$ sci_ a r t e x t \& p i d = S 0213 $12852004000400003 \& \operatorname{lng}=$ es. http://dx.doi.org/10.4321/S021312852004000400003.
2. Méndez Alburez LP. Medición del grado de ansiedad en odontólogos generales, respecto a la atención de pacientes niños en la práctica privada [tesis de grado]. Guatemala. Universidad San Carlos. 2004. Disponible en: [http://radiou.usac. edu.gt/fdeo/biblio/boletines/alerta1_2006.pdf]

3. Locker D, Liddell A, Shapiro D. Diagnostic categories of dental anxiety: a population-based study.
Behaviour Research and Therapy. 1999;(37):25-37.

4. Márquez Rodríguez, JA y col. ¿Por qué se le tiene miedo al dentista? Estudio descriptivo de la posición de los pacientes de la Sanidad Pública en relación a diferentes factores subyacentes a los miedos dentales. RCOE. 2004 [citado2012Ene]; 9(2):165-174. Disponible en: http://scielo.isciii.es/pdf/rcoe/ v9n2/original3.pdf 